\title{
NITROGEN CONCENTRATION ESTIMATION WITH HYPERSPECTRAL LIDAR
}

\author{
O. Nevalainen ${ }^{\text {a, } * \text {, T. Hakala }}{ }^{\text {, }}$ J. Suomalainen ${ }^{\text {a, b }}$, S. Kaasalainen ${ }^{\text {a }}$
}

\author{
${ }^{a}$ Department of Photogrammetry and Remote Sensing, Finnish Geodetic Institute, Geodeetinrinne 2, 02431, Masala, \\ Finland - (olli.nevalainen, teemu.hakala, juha.suomalainen, sanna.kaasalainen)@fgi.fi \\ ${ }^{\mathrm{b}}$ Geo-Information Science and Remote Sensing, Wageningen University, 6700 AA Wageningen, The Netherlands
}

KEY WORDS: Remote sensing, Hyperspectral, LiDAR, Laser scanning, Nitrogen estimation

\begin{abstract}
:
Agricultural lands have strong impact on global carbon dynamics and nitrogen availability. Monitoring changes in agricultural lands require more efficient and accurate methods. The first prototype of a full waveform hyperspectral Light Detection and Ranging (LiDAR) instrument has been developed at the Finnish Geodetic Institute (FGI). The instrument efficiently combines the benefits of passive and active remote sensing sensors. It is able to produce 3D point clouds with spectral information included for every point which offers great potential in the field of remote sensing of environment. This study investigates the performance of the hyperspectral LiDAR instrument in nitrogen estimation.

The investigation was conducted by finding vegetation indices sensitive to nitrogen concentration using hyperspectral LiDAR data and validating their performance in nitrogen estimation. The nitrogen estimation was performed by calculating 28 published vegetation indices to ten oat samples grown in different fertilization conditions. Reference data was acquired by laboratory nitrogen concentration analysis. The performance of the indices in nitrogen estimation was determined by linear regression and leave-one-out cross-validation.

The results indicate that the hyperspectral LiDAR instrument holds a good capability to estimate plant biochemical parameters such as nitrogen concentration. The instrument holds much potential in various environmental applications and provides a significant improvement to the remote sensing of environment.
\end{abstract}

\section{INTRODUCTION}

Agricultural lands are major carbon sinks on Earth and they have strong impact on global carbon dynamics and nitrogen availability. In addition, monitoring crop physiological status, such as nitrogen balance and biomass, is essential for precision agriculture and farm management (Haboudane et al., 2008). Hence, efficient and accurate methods are needed to detect and estimate the key processes in agro-ecosystems.

Passive multi- and hyperspectral remote sensing offer efficient methods of producing information about vegetation spectral properties, which is needed for studying vegetation biochemical parameters, such as nitrogen concentration. However, some notable limitations still exist with passive remote sensing techniques. Passive sensors are dependent on solar illumination and they are sensitive to changing illumination conditions (Nischan et al., 2003; Puttonen et al, 2010). In addition, they provide no proper information on the vertical distribution of vegetation, which is essential in vegetation biomass estimation (Jones et al., 2010; Woodhouse et al., 2011).

Light detection and ranging (LiDAR) is a powerful tool for acquiring information on vegetation structure and biomass. LiDAR sensors are capable of penetrating the vegetation canopy and thus receive measurement data from both the canopy and sub-canopy structures (Lim et al., 2003; Tan et al., 2004; Jones et al., 2010; Woodhouse et al., 2011). LiDAR has been widely utilized, for example, in retrieving various forest parameters such as the tree height and stem diameter (Hyyppä et al., 2001). Another major benefit of LiDAR sensors is the capability to operate day or night and they are not affected by changing illumination conditions (Nischan et al., 2003).

The disadvantage of LiDAR in vegetation measurements has been the lack of spectral information (Lim et al., 2003; Wallace et al., 2012). Most laser scanners operate at a single-wavelength providing point data with a high spatial resolution and intensity data. The use of laser intensity in target and surface classification has been a topic of interest (Moffiet et al., 2005; Kaasalainen et al., 2008). However, the single-wavelength laser intensity data does not provide spectral data to enable, for example, estimation of vegetation biochemical parameters. Therefore, a multi-wavelength active measurement system has been an object of great interest (Puttonen et al., 2010; Wallace et al., 2012).

The benefits of passive and LiDAR sensors in vegetation remote sensing have led to several attempts to combine these techniques in one measurement system (Johnson et al., 1999; Tan et al., 2004; Chen et al., 2010; Woodhouse et al., 2011; Wallace et al., 2012; Wei et al., 2012). LiDAR and passive sensor data has shown to complement each other very well (Koetz et al., 2007; Jones et al., 2010). However, combining two data sets acquired with different types of instruments cause significant spatial and temporal registration problems.

Another approach has been to develop a multi-wavelength LiDAR instrument. Previous research has mainly focused on dual-wavelength LiDAR systems (Tan et al., 2004; Chen et al., 2010; Wallace et al., 2012) but LiDAR system operating with more than two wavelengths has also been developed

\footnotetext{
* Corresponding author.
} 
(Woodhouse et al., 2011; Wei et al., 2012). These previous approaches have shown the potential for multi-wavelength LiDAR systems in vegetation remote sensing. However, the wavelength range of available spectral bands and the number of sensor channels has been quite limited in these approaches, because they use separate monochromatic lasers as light sources.

The development of an active hyperspectral LiDAR instrument has become possible since the development and the commercial availability of supercontinuum laser sources (Kaasalainen et al., 2007). A supercontinuum laser source can produce directional broadband light which in combination with a hyperspectral time-of-flight sensor constitutes an instrument capable of simultaneous distance and broadband reflectance measurement (Hakala et al., 2012). An instrument utilizing the supercontinuum laser technique has been developed and constructed at the Finnish Geodetic Institute (FGI). The instrument is the first prototype of a full waveform terrestrial hyperspectral LiDAR (Hakala et al., 2012). It can measure 3D point clouds included with hyperspectral backscattered reflectance (Kaasalainen et al., 2010) for every point. The reflectance data is collected with eight channels ranging between 500-1000 $\mathrm{nm}$. The hyperspectral LiDAR instrument combines the benefits of both passive and active sensors in a single instrument thus enabling it to extract important biochemical and structural parameters of vegetation in one measurement.

Plant nitrogen concentration is strongly linked to plant chlorophyll concentration. Chlorophylls are an important indicator of plant photosynthetic capacity, productivity and stress level (Blackburn et al., 1998; Zhang et al., 2008). The amount of chlorophyll in plants decreases while plant is under stress (Zhang et al., 2008). The chlorophylls a and b have a strong reflectance peak in the green region (around $550 \mathrm{~nm}$ ) and strong absorbance peaks in the visible red $(630-690 \mathrm{~nm})$ and blue $(450-520 \mathrm{~nm})$ regions of the vegetation spectrum. Consequently, variations in vegetation chlorophyll levels cause significant spectral changes especially at the red-edge region which is the most important and investigated part of the vegetation spectrum (Main et al., 2011). The spectral red-edge is an abrupt change in reflectance between the red $(670 \mathrm{~nm})$ and near-infrared (NIR, $800 \mathrm{~nm}$ ) regions, caused by combined effects of chlorophyll absorption in the red region and high reflectance of vegetation in the NIR-region.

The most common method to detect variations in the vegetation spectral red-edge and nitrogen and chlorophyll levels has been to define various vegetation indices from remotely sensed data. Vegetation indices are based on combinations of reflectance at certain wavelengths that highlight important features in the vegetation reflectance spectrum.

This research investigates the abilities of hyperspectral LiDAR in vegetation remote sensing. The investigation is conducted by finding vegetation indices sensitive to nitrogen concentration of Oats (Avena Sativa) grown in different fertilization conditions using hyperspectral LiDAR data and validating their performance in nitrogen estimation. The results of the estimation using hyperspectral LiDAR data are compared with nitrogen concentration analysis performed in a laboratory. The comparison is carried out by linear regression and leave-one-out cross-validation.

\section{MATERIALS AND METHODS}

\subsection{Oat samples}

The oat (Avena sativa) samples were grown in ten $400 \times 300 \mathrm{x}$ $120 \mathrm{~mm}$ plastic trays outside in Masala, Kirkkonummi, Finland. The oat trays were seeded at the end of May of 2012. The trays were seeded using two different seeding densities and five different fertilization levels. The used seeding densities were approximately 21 and $42 \mathrm{~g} / \mathrm{m}^{2}$. The used fertilization levels were $0,4.5,12,18$ and $27 \mathrm{gN} / \mathrm{m}^{2}$ (gram of nitrogen per square meter). The surface area of the trays was $0.12 \mathrm{~m}^{2}$. The oat samples were grown for six weeks before they were measured.

\subsection{Hyperspectral LiDAR instrument}

The instrument used in this research is the prototype of a full waveform terrestrial hyperspectral LiDAR instrument presented in Hakala et al. (2012), but with different laser source. The laser source used in the current instrument configuration is LEUKOS-SM (LEUKOS). It is capable of producing $1 \mathrm{~ns}$ laser pulses ranging approximately from 400 to $2200 \mathrm{~nm}$ with a pulse rate of $5 \mathrm{kHz}$. The power of the laser pulses is approximately $200 \mathrm{~mW}$. It can measure 3D point clouds included with hyperspectral backscattered reflectance for every point. For more details on the instrument operation principles, calibration and waveform processing see Hakala et al. (2012).

\subsection{Hyperspectral LiDAR measurements}

The oat samples were scanned individually from two different scanning directions. First, the samples were scanned from a nadir point of view (incidence angle $=0^{\circ}$ ) and then from the side with an incidence angle of approximately 75 degrees. The nadir scan was attained by placing a mirror above the oat sample. During the side scan configuration a black matte canvas was placed behind the scanned sample to minimize background reflections. The scanning ranges using the nadir and side scan geometry were approximately 5.3 and $4.3 \mathrm{~m}$ respectively.

The oat samples were measured using eight spectral channels which had channel centers at 554.8, 623.5, 691.1, 725.5, 760.3, 795.5, 899.0 and $1000.4 \mathrm{~nm}$ and Full Width at Half Maximum (FWHM) approximately $18.5 \mathrm{~nm}$. The horizontal resolution used in the nadir and side scan configuration was 0.05 and 0.1 degrees respectively. The number of vertical scan lines was 40 using the nadir scan and 60 while scanning from the side. To ensure the quality of the nadir scan measurements, a Spectralon (Labsphere Inc.) reference plate was scanned for calibration purposes.

\subsection{Nitrogen concentration acquisition}

After the oats were measured with the hyperspectral LiDAR, they were transported to laboratory analysis in dark and cool conditions. The nitrogen concentration analysis was performed in the Agrifood Research Finland (MTT) in Jokioinen, Finland. The analysis was performed using the Kjeldahl method (Lynch \& Barbano, 1999). The MTT laboratory provided the nitrogen concentration of the oat samples as a percentage of the amount of nitrogen in fresh weight. The extended uncertainty of the laboratory measurements was $\pm 15 \%$. 


\subsection{Hyperspectral LiDAR data processing}

Noise points and measurement points from non-photosynthetic materials, such as the plastic tray, background canvas and soil, were removed from the measured point clouds. After removing these points, point clouds produced using the side scan configuration consisted of approximately 20000 points and point clouds measured using the nadir scan configuration had approximately 19500 points in the low seeding density oats and 31000 with high seeding density oats.

\subsection{Estimation method}

The nitrogen concentration estimation was performed by finding vegetation indices sensitive to oat nitrogen concentration using hyperspectral LiDAR data and validating their performance in nitrogen estimation. 28 published vegetation indices related to chlorophyll and nitrogen concentration were tested. The mean of vegetation index value calculated for every point in the point cloud was used as the estimator for nitrogen concentration.

The best result with the side scan data was achieved with the Chlorophyll Absorption Ratio Index (CARI), whereas the Normalized Difference Red Edge (NDRE) provided the best result with nadir scan data. CARI was designed by Kim et al (1994) to reduce the variability of the photosynthetically active radiation caused by diverse non-photosynthetic materials (Haboudane et al., 2002). It uses reflectance at 550 and $700 \mathrm{~nm}$, which correspond to the minimum absorption of the photosynthetic pigments together with reflectance at the maximum absorbance of chlorophyll-a around $670 \mathrm{~nm}$. CARI is calculated using the following equation:

$$
C A R I=\frac{R_{700}}{R_{670}} * \frac{\sqrt{\left(670 a+R_{670}+b\right)^{2}}}{\sqrt{\left(a^{2}+1\right)}},
$$

where

$$
\begin{aligned}
& a=\left(R_{700}-R_{550}\right) / 150 \\
& b=R_{550}-550 a
\end{aligned}
$$

Hyperspectral LiDAR instrument channels $3(691 \mathrm{~nm}), 2$ (623 $\mathrm{nm})$ and $1(554 \mathrm{~nm})$ were selected to correspond to reflectance bands R700, R670 and R550 respectively.

NDRE was presented by Barnes et al. (2000) to be used as a vegetation stress indicator with Canopy Chlorophyll Content Index (CCCI). NDRE is designed to respond to changes in chlorophyll concentration and nitrogen content (Rodriguez et al., 2006; Tilling et al, 2007). The equation of NDRE is:

$$
N D R E=\frac{R_{790}-R_{720}}{R_{790}+R_{720}}
$$

Hyperspectral LiDAR instrument channels $6(795 \mathrm{~nm})$ and 4 (725 nm) were selected to correspond to reflectance bands R790 and R720 respectively.

\subsection{Validation methods}

The performance of the vegetation indices in nitrogen concentration estimation was determined with linear regression analysis and leave-one-out cross-validation (LOOCV). The performance of the linear regression fit was evaluated with the coefficient of determination $\left(\mathrm{R}^{2}\right)$ and LOOCV root mean square error (RMSE).

The leave-one-out method is based on using a single observation as the validation data while the remaining observations are used as the training data. The process is repeated until every observation is used once as the validation set. The LOOCV gives deeper insight to the dataset and fit of the model even with relatively small datasets such as in this study. The advantage of LOOCV method is that every observation is used in training and validating the model.

\section{RESULTS AND DISCUSSION}

The objective of growing the oats with varying fertilization levels and seeding densities was to have oats with clearly varying nitrogen concentrations. The objective was not achieved as well as it was intended as the nitrogen concentrations were varying but the variations between the oats were quite small, especially with the low density (L1-L5) oats (Table 1). The nitrogen concentrations in the low density oats varied between 5.10 and $5.80(\mathrm{mg} / \mathrm{g})$ with oats $\mathrm{L} 1$ and $\mathrm{L} 2$ having the same concentration of $5.10 \mathrm{mg} / \mathrm{g}$. In addition, the highest nitrogen concentration in the low density oats was measured in the L1 oat which was assumed to have the lowest nitrogen concentration. The relatively high nitrogen concentration of the L1 oat might be explained by the loss of other substances within the oat. The L1 oat had already stopped growing and become yellowish due to the lack of nutrients. However, a better distribution of nitrogen concentrations was achieved with the high density oats $\mathrm{H} 1-\mathrm{H} 5$, which had nitrogen concentration levels varying between 3.90 and $6.00 \mathrm{mg} / \mathrm{g}$.

\begin{tabular}{cc}
\hline Oat number & $\begin{array}{c}\text { Nitrogen concentration } \\
(\mathbf{m g} / \mathbf{g})\end{array}$ \\
\hline L1 & 5.80 \\
L2 & 5.10 \\
L3 & 5.10 \\
L4 & 5.40 \\
L5 & 5.70 \\
H1 & 3.90 \\
H2 & 4.70 \\
H3 & 4.80 \\
H4 & 5.00 \\
H5 & 6.00 \\
\hline
\end{tabular}

Table 1 Laboratory analysed nitrogen concentration of oat samples provided by MTT.

The lack of variation in the nitrogen concentrations induced that there were not significant differences between the reflectance spectra of the side scanned oats configuration (Figure 1). Especially, the differences of the low density oats in the chlorophyll absorption region at 630-690 nm were small. However, differences in the low density oats can be seen in the NIR region, due to structural differences in the oat leaf foliage. The oats with denser leaf foliage have higher reflectance at NIR. The high density oats which had better distribution of nitrogen concentrations show visible differences even around 630-690 nm. 

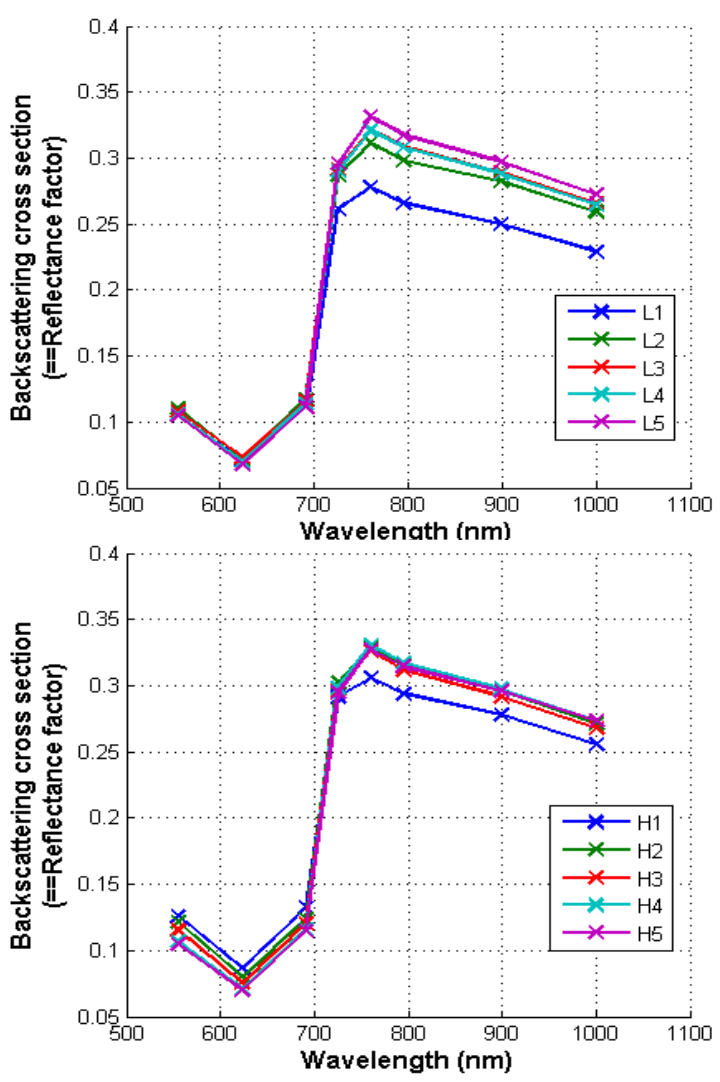

Figure 1 Average reflectance spectra of the side scanned oat samples. L indicating low and $\mathrm{H}$ high seeding density and the numbers from one to five indicate fertilization level with five being the highest fertilization level.

The nadir scanned oat reflectance spectra show much more variation between the oats than the side scanned ones. The oat reflectance spectra from the nadir scan data are presented in Figure 2. The strong differences in the reflectance spectra of the side and nadir scan data is an outcome of the different scanning geometries. The main difference is that using the side scan configuration there is not any soil background affecting the measurements. In addition, the nadir scan configuration produces more measurements from the oat canopy top which has more large leaves, whereas the side scan configuration produces more measurements from the stem parts of the oats. Consequently, the variations in the oat spectra in the nadir scan case are mainly due to the structural differences in the leaf canopies, such as the density and size of the leaves. When the leaf canopy is denser, there is stronger backscattered reflectance from the leaves, especially at NIR region, and less background effect from the soil.

The best performance with side scan configuration was acquired using CARI $\left(\mathrm{R}^{2}=0.85, \mathrm{RMSE}=0.229 \mathrm{mg} / \mathrm{g}\right.$, RMSE leave-oneout $=0.267 \mathrm{mg} / \mathrm{g}$ ). The regression plot of CARI between the oat nitrogen concentrations is presented in Figure 3. The best performance using the nadir scan configuration was achieved with NDRE $\left(\mathrm{R}^{2}=0.76\right.$, RMSE leave-one-out $\left.=0.374 \mathrm{mg} / \mathrm{g}\right)$. The regression plot of NDRE is presented in Figure 4. NDRE has previously shown sensitivity to leaf nitrogen content, for example, in Rodriguez et al. (2006) where $\mathrm{R}^{2}$ of 0.71 was achieved while estimating nitrogen content in wheat (Triticum aestivum) using a portable spectrometer.
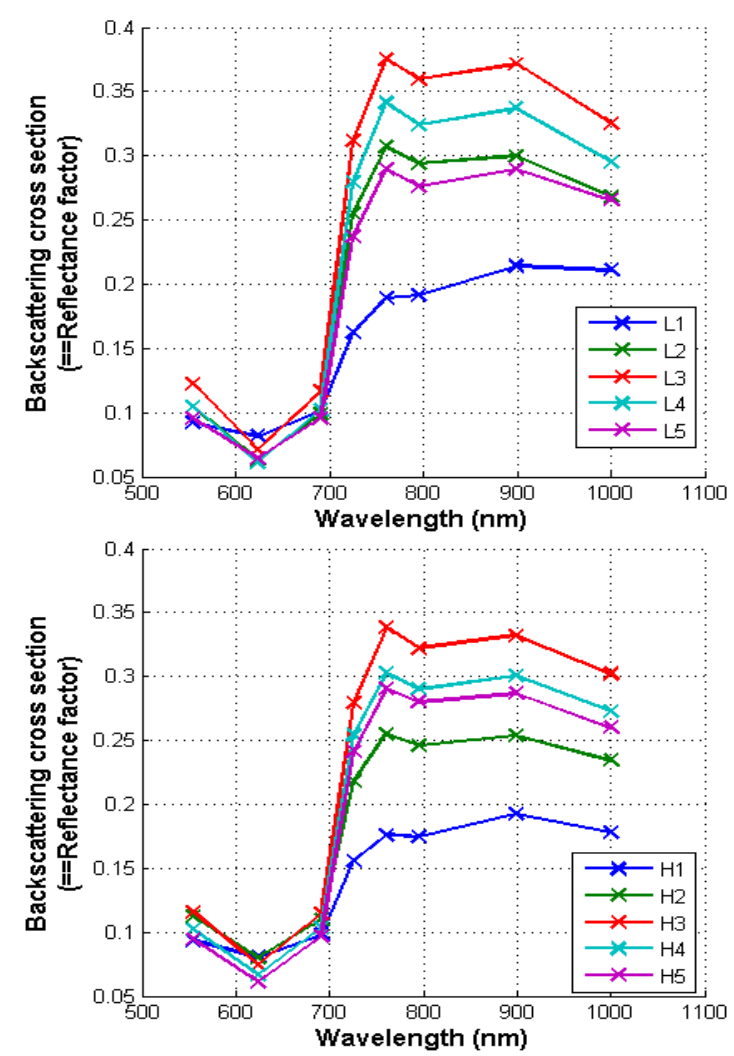

Figure 2 Average reflectance spectra of the nadir scanned oat samples. $\mathrm{L}$ indicating low and $\mathrm{H}$ high seeding density and the numbers from one to five indicate fertilization level with five being the highest fertilization level.

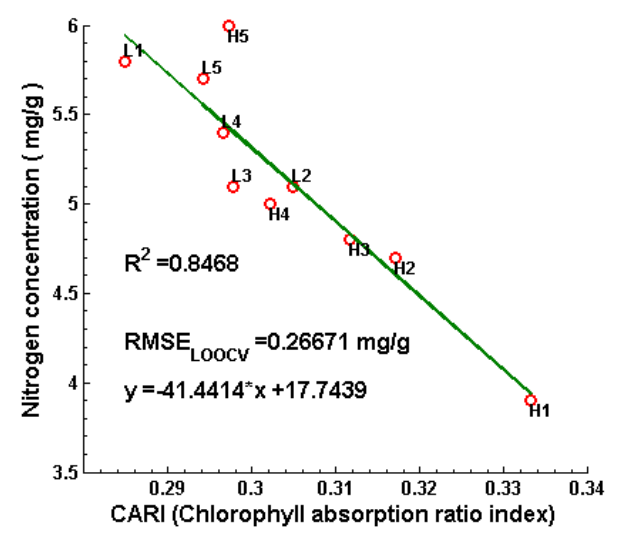

Figure 3 Regression plot between the laboratory analysed nitrogen concentration $(\mathrm{mg} / \mathrm{g})$ and the Chlorophyll Absorption Ratio Index (CARI).

The results indicate that some vegetation indices, such as CARI and NDRE, derived from hyperspectral LiDAR data are able to estimate nitrogen concentration in oats relatively well. The weaker performance of some indices might be a result of small dataset with the oats having small variation in nitrogen concentrations but high variation in leaf canopy structures. The interference, possibly caused by the mirror, in the nadir scan configuration might have also influenced the results. 


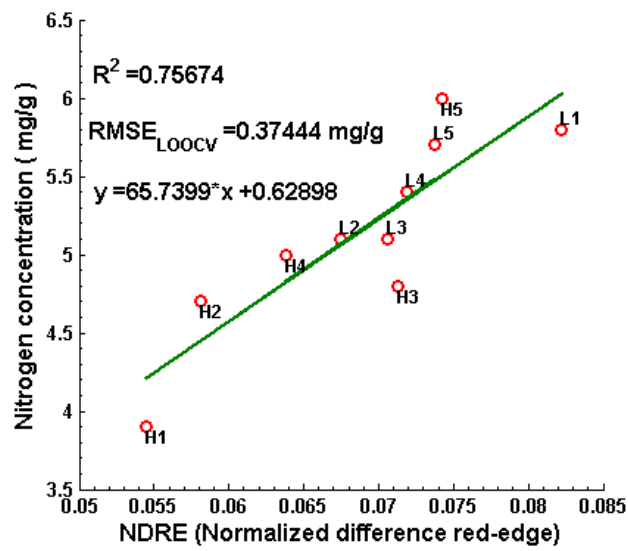

Figure 4 Regression plot between the laboratory analysed nitrogen concentration $(\mathrm{mg} / \mathrm{g})$ and the Normalized Differential Red-edge (NDRE).

The remote sensing based nitrogen estimation using wavelengths in $550-1000 \mathrm{~nm}$ region is based on detecting changes induced by chlorophyll absorption in the vegetation reflectance spectrum. Nitrogen, however, is included also in other substances within plants and thus amount of nitrogen might not be estimated as efficiently as chlorophylls with vegetation indices using chlorophyll sensitive wavelengths. For example, much of the nitrogen is stored in proteins which have main absorption regions at 1500, 1680, 1740, 1940, 2050, 2170, 2290 and $2470 \mathrm{~nm}$ (Kumar et al., 2001) which were out of the spectral range of the hyperspectral LiDAR instrument.

There was variability among the performances of the tested vegetation indices in nitrogen estimation. In addition, there were differences in the performances of the indices when using different scanning geometries. Some indices showed poor correlation to nitrogen concentration probably due to the indices being insensitive to the structural and spectral properties of the oats or incompatible with the reflectance values of the hyperspectral LiDAR instrument channels. Vegetation indices are sensitive to the to the measured species and used sensor properties which has to be considered when comparing results obtained with the vegetation indices used in this study to other studies using a different type of sensor and plant species as test subjects.

It must be noted that nitrogen concentration was estimated using wavelengths and vegetation indices related to chlorophylls. Although chlorophyll and nitrogen concentration are linked to each other, better results might have been achieved by performing chlorophyll estimation by assaying chlorophyll concentration of the oats instead of nitrogen concentration. Widening the spectral range of the hyperspectral LiDAR instrument to $1500 \mathrm{~nm}$, where a known nitrogen absorption band is located, could also improve the capabilities of the instrument to estimate nitrogen concentration.

The dataset used in this research consisted only of ten specimens. A larger dataset would have provided more diversity and variance to the analysis providing more reliability to the results. More precise validation of hyperspectral LiDAR in nitrogen concentration estimation would require larger dataset with much more variability within concentration levels and species.
A number of previous studies concentrated on nitrogen estimation by remote sensing have concentrated on estimating the nitrogen content per unit area $\left(\mathrm{mg} / \mathrm{m}^{2}\right)$ instead of concentration $(\mathrm{mg} / \mathrm{g})$ as was done in this research. Further study is, hence, recommended to investigate whether the results would be any different if nitrogen content had been estimated instead of nitrogen concentration.

A subject of future studies could also be the use of inversion of radiative models, such as PROSPECT (Leaf Optical Properties Spectra) and LIBERTY (Leaf Incorporating Biochemistry Exhibiting Reflectance and Transmittance Yields). By using model inversion in nitrogen estimation some of the limitations of vegetation indices might be overcome.

\section{CONCLUSION}

The purpose of this research was to investigate capability of the first prototype of a full waveform terrestrial hyperspectral LiDAR in nitrogen estimation. The nitrogen estimation was performed by calculating vegetation indices to oats grown in different fertilization conditions. Linear regressions were performed with the calculated indices and laboratory analyzed concentrations. The results indicated that few vegetation indices derived from hyperspectral LiDAR data were able to form relatively good linear relationship with nitrogen concentration of the oats. The best performance was acquired using CARI (Chlorophyll absorption ratio index) with a coefficient of determination of 0.85 . The results indicate that hyperspectral LiDAR offers great benefits to the remote sensing of vegetation.

In the near future, the further development of the hyperspectral LiDAR instrument includes adding mobility, enhancing the spectral and range capabilities and improving the eye safety. The instrument already holds much potential in various environmental applications. As the hyperspectral LiDAR technology matures, operational mobile and airborne hyperspectral laser scanners will be available from commercial manufacturers and provide significant improvement to the remote sensing of environment.

\section{REFERENCES}

Barnes, E., Clarke, T. \& Richards, S., 2000. Coincident detection of crop water stress, nitrogen status and canopy density using ground based multispectral data. In: Proceedings of the 5th International Conference on Precision Agriculture, Bloomington, MN, USA.

Blackburn, G., 1998. Quantifying chlorophylls and carotenoids from leaf to canopy scales: An evaluation of some hyperspectral approaches. Remote Sensing of Environment, 66, pp. 273-285.

Chen, Y., Räikkönen, E., Kaasalainen, S., Suomalainen, J., Hakala, T., Hyyppä, J. \& Chen, R., 2010. Two-channel Hyperspectral LiDAR with a Supercontinuum Laser Source. Sensors, 10(7), pp. 7057-7066.

Haboudane, D., Miller, J., Tremblay, N., Zarco-Tejada, P. \& Dextraze, L,. 2002. Integrated narrow-band vegetation indices for prediction of crop chlorophyll content for application to precision agriculture. Remote Sensing of Environment, 81(2-3), pp. 416-426. 
Haboudane, D.; Tremblay, N.; Miller, J. \& Vigneault, P., 2008. Remote Estimation of Crop Chlorophyll Content Using Spectral Indices Derived From Hyperspectral Data. Geoscience and Remote Sensing, IEEE Transactions on, 46(2), pp.423-437

Hakala, T., Suomalainen, J., Kaasalainen, S. \& Chen, Y., 2012. Full waveform hyperspectral LiDAR for terrestrial laser scanning. Optical Express, 20, pp. 7119-7127.

Hyyppä, J., Kelle, O., Lehikoinen, M. \& Inkinen, M., 2001. A segmentation-based method to retrieve stem volume estimates from 3-D tree height models produced by laser scanners. Geoscience and Remote Sensing. IEEE Transactions on, 39(5), pp. 969-975.

Johnson, B., Joseph, R., Nischan, M., Newbury, A., Kerekes, J., Barclay, H., Willard, B. \& Zayhowski, J., 1999. Compact active hyperspectral imaging system for the detection of concealed targets. Proceedings of SPIE, 3710, pp. 144-153.

Jones, T., Coops, N. \& Sharma, T., 2010. Assessing the utility of airborne hyperspectral and LiDAR data for species distribution mapping in the coastal Pacific Northwest, Canada. Remote Sensing of Environment, 114(12), pp. 2841-2852.

Kaasalainen, S., Lindroos, T. \& Hyyppä, J., 2007. Toward Hyperspectral Lidar: Measurement of Spectral Backscatter Intensity With a Supercontinuum Laser Source. Geoscience and Remote Sensing Letters IEEE, 4(2), pp. 211-215.

Kaasalainen, S., Kukko, A., Lindroos, T., Litkey, P., Kaartinen, H., Hyyppä, J. \& Ahokas, E., 2008. Brightness Measurements and Calibration With Airborne and Terrestrial Laser Scanners. Geoscience and Remote Sensing, IEEE Transactions on, 46(2), pp. 528-534.

Kaasalainen, S., Niittymaki, H., Krooks, A., Koch, K., Kaartinen, H., Vain, A. \& Hyyppa, H., 2010. Effect of Target Moisture on Laser Scanner Intensity. Geoscience and Remote Sensing, IEEE Transactions, 48(4), pp. 2128-2136.

Kim, M., Daughtry, C., Chappelle, E., Mcmurtrey, J. \& Walthall, C., 1994. The use of high spectral resolution bands for estimating absorbed photosynthetically active radiation (A par). Proceedings of 6th International Symposium on Physical Measurements and Signatures in Remote Sensing, pp. 299-306.

Koetz, B., Sun, G., Morsdorf, F., Ranson, K., Kneubühler, M., Itten, K. \& Allgöwer, B., 2007. Fusion of imaging spectrometer and LIDAR data over combined radiative transfer models for forest canopy characterization. Remote Sensing of Environment, 106(4), pp. 449-459.

Kumar, L., Schmidt, K., Dury, S. \& Skidmore, A., 2001. Imaging spectrometry and vegetation science. In F. van de Meer and S.M. de Jong (Eds). Imaging Spectrometry, Kluwer Academic Press: Dordrecht, pp. 111-155.

Lim, K., Treitz, P., Wulder, M., St-Onge, B. \& Flood, M., 2003. LiDAR remote sensing of forest structure. Progress In Physical Geography, 27(1), pp. 88-106.

Lynch, J. \& Barbano, D., 1999. Kjeldahl nitrogen analysis as a reference method for protein determination in dairy products. Journal of AOAC International, 82(6), pp. 1389-1398.
Main, R., Cho, M., Mathieu, R., O’Kennedy, M., Ramoelo, A. \& Koch, S., 2011. An investigation into robust spectral indices for leaf chlorophyll estimation. ISPRS Journal of Photogrammetry and Remote Sensing, 66(6), pp. 751-761.

Moffiet, T., Mengersen, K., Witte, C., King, R. \& Denham, R., 2005. Airborne laser scanning: Exploratory data analysis indicates potential variables for classification of individual trees or forest stands according to species. ISPRS Journal of Photogrammetry and Remote Sensing, 59(5), pp. 289-309.

Nischan, M., Joseph, R., Libby, J. \& Kerekes, J., 2003. Active Spectral Imaging. Lincoln Laboratory Journal, Special Issue on Spectral Imaging, 14(1), pp. 131-144.

Puttonen, E., Suomalainen, J., Hakala, T., Räikkönen, E., Kaartinen, H., Kaasalainen, S. \& Litkey, P., 2010. Tree species classification from fused active hyperspectral reflectance and LIDAR measurements. Forest Ecology and Management, 260(10), pp. 1843-1852.

Rodriguez, D., Fitzgerald, G., Belford, R. \& Christensen, L., 2006. Detection of nitrogen deficiency in wheat from spectral reflectance indices and basic crop eco-physiological concepts. Australian Journal of Agricultural Research, 57, pp. 781-789

Tan, S. \& Narayanan, R., 2004. Design and Performance of a Multiwavelength Airborne Polarimetric Lidar for Vegetation Remote Sensing. Applied Optics, 43, pp. 2360-2368.

Tilling, A., O’Leary, G., Ferwerda, J., Jones, S., Fitzgerald, G., Rodriguez, D. \& Belford, R., 2007. Remote sensing of nitrogen and water stress in wheat. Field Crops Research, 104(1-3), pp. $77-85$.

Wallace, A., Nichol, C. \& Woodhouse, I., 2012. Recovery of Forest Canopy Parameters by Inversion of Multispectral LiDAR Data. Remote Sensing, 4(2), pp. 509-531.

Wei, G., Shalei, S., Bo, Z., Shuo, S., Faquan, L. \& Xuewu, C., 2012. Multi-wavelength canopy LiDAR for remote sensing of vegetation: Design and system performance. ISPRS Journal of Photogrammetry and Remote Sensing, 69, pp. 1-9.

Woodhouse, I., Nichol, C., Sinclair, P., Jack, J., Morsdorf, F., Malthus, T. \& Patenaude, G., 2011. A Multispectral Canopy LiDAR Demonstrator Project. Geoscience and Remote Sensing Letters, IEEE, 8(5), pp. 839-843

Zhang, Y., Chen, J., Miller, J. \& Noland, T., 2008. Leaf chlorophyll content retrieval from airborne hyperspectral remote sensing imagery. Remote Sensing of Environment, 112(7), pp. 3234-3247.

\section{ACKNOWLEDGEMENTS}

This research was funded by the Academy of Finland research projects "New techniques in active remote sensing: hyperspectral laser in environmental change detection" and "Mobile hyperspectral laser remote sensing".

The authors would like to thank Outi Kurri and Merja Uusitupa in MTT for the nitrogen concentration analysis. 